\title{
Data-Driven Business Opportunity Decision Making Using Markov Chain Model
}

\author{
T Abiram*, PSS Anudeep, S Mahesh, T Ramanjaneyulu \\ Kallam Haranadhareddy Institute of Technology, India
}

*Corresponding author: $\mathrm{T}$ Abiram, Kallam Haranadhareddy Institute of Technology, Guntur, India.
Received Date: May 12, 2020

Published Date: May 29, 2020

\begin{abstract}
Business opportunity assessment with an information driven method may not be a simple or direct procedure. The accomplishment of the business opportunity is dependent upon outer market factors, including economic situations for businesspeople when all is said in done and administrative systems influencing access to customer and work markets and fund. The business visionary may have a constrained or no influence over such outer conditions. In this way, the data stream important to finish up the monetary standpoint of the market (positive or negative) for the business opportunity may not be accessible (i.e., the market data might be defective). Besides, the "genuine market" possibly shrouded when that data isn't noticeable. This undertaking intends to build up an algorithmic information examination method for business visionaries to break down inadequate information from outer market factors for a speculation portfolio under the information driven point of view of enterprise. The ideal speculation portfolio adjusts the exchange off between expected returns and market data and is furtherly directed by the businessperson's hazard inclination and operational deficiencies. The proposed somewhat watched Markov choice procedure (POMDP) model sets up a probabilistic connection between a noticeable and the shrouded advertise process by means of the business visionary's venture portfolio. At that point the model recognizes a probabilistic data measure to evaluate the association between the perceptible and undetectable procedure to all the more likely comprehend the condition of the market for the business opportunity. With the probabilistic data measure, the business visionary at that point could develop and test new speculation portfolios that are most appropriate for the market concerning businessperson hazard inclination and operational deficiencies.
\end{abstract}

Keywords: Business opportunity evaluation; Decision making under uncertainty; Partially observed Markov decision process

\section{Introduction}

The term businessperson is utilized to allude to the organizer of the new business, or the individual who began the new market where there was none previously. The individual does innovative activities that lead to the development of another firm. The business visionary is somebody who represents considerable authority in assuming liability for and settling on vital choices about the item, the nature of the association, its area, the requirement for capital, and different assets. A businessperson is somebody who can distinguish a need in the market, give an item or administration to address that issue, and, in that procedure, win a benefit.

The word businessperson showed up in the French language sometime before the development of the idea of pioneering capacity. Richard Cantillon recognized a businessperson's one of a kind hazard bearing component in the mid-eighteenth century. John Stuart Mill promoted the term business visionary in his 1884 book, Principles of Political Economy. Definitions from the universe of financial aspects vary from those in the realm of the board. There are contrasts among businesspeople, and administrators' enterprise is a method of dealing with that includes seeking after circumstance regardless of the assets at present controlled. Business visionaries recognize opportunity, gather required assets, execute a sensible activity plan, and reap the prize in a hopeful, adaptable way. According to them, a Capitalist was one who accepted the hazard or vulnerability, while a businessperson got and composed creation variables to make esteem.

The term business visionary, which means somebody who spent significant time in sorting out business exercises. Understanding the 
nature and wellsprings of vulnerability that underlie enterprising dynamic has been an essential focal point of business enterprise inquire about. The imbuement of information investigation methods (i.e., reviewing, changing, and demonstrating information to help dynamic) and advances (e.g., information examination) in business has produced better approaches for managing vulnerability. For instance, the steady progression of "enormous information" obtained through web-based life applications (e.g., Twitter) has been dissected to conquer opportunity-related weaknesses in social insurance. A developing number of financial speculators have utilized computerized information investigation methods to assess business ventures. We allude to the pattern of information driven methods and innovations in forming exercises of the pioneering procedure (i.e., opportunity acknowledgment, advancement, and assessment) as "information driven business."

In any case, business opportunity assessment with an information driven strategy may not be a simple or direct procedure. The accomplishment of the business opportunity is dependent upon outside market factors, including economic situations for businesspeople all in all and administrative systems influencing access to customer and work markets and fund. The businessperson may have a constrained or no power over such outer conditions. In this manner, the data stream important to finish up the monetary standpoint of the market-positive or negative - for the business opportunity may not be accessible (i.e., the market data might be defective). Besides, the "genuine market" perhaps concealed when that data isn't noticeable. For instance, in the growing clean vitality market of Turkey, the administration's blended signals on a feed-in-tax (an administration arrangement component meant to quicken interest in clean vitality) made it hard for business visionaries to confront the lack of assets to assess the possibilities of the chance. As the information driven viewpoint of business enterprise recommends a powerful innovative procedure we build up an algorithmic information examination method for the business visionaries to process fragmented information and uncover the shrouded idea of the market over a specific period. Eminently, every episode of an operational deficiency in our model perceives an alternate level of effect on the business visionary's assessment of the condition of the market for the business opportunity (i.e., to what degree the market underpins the business opportunity). The businessperson's individual hazard inclination further conservatives the level of that sway. Our findings expand the writing on pioneering dynamic and information driven enterprise by looking at how the business visionary's hazard inclination and operational deficiencies shape the business visionary's chance related assessments and choices in a dynamic and persistently developing condition. As indicated by our numerical outcomes, the business visionary may pick a speculation portfolio, subject to his/ her degrees of money and hazard inclination, that would permit a hazard supporting or market- controlling technique instead of looking for just high anticipated returns. Practically speaking, our POMDP-based model gives business visionaries and supervisors an algorithmic information examination procedure to utilize bits of knowledge from information investigation for assessing the engaging quality of a business opportunity.

\section{Literature Survey}

This writing audit portrays how our examination adds to the writing on pioneering dynamic (especially for assessing a business opportunity), operational enterprise, information driven viewpoint of enterprise, and ideal portfolio interest in incompletely watched Markov chains.

Opportunity assessment is the center of pioneering dynamic. Enterprise researchers have broadly explored how businesspeople settle on circumstance assessment choices dependent on singular variables (e.g., comprehension and desires) joined with outside elements (e.g., valuation of the market) (see Shepherd et al. (2015); Short et al. (2010) for surveys). Mc Kelvey et al. McKelvey et al. [1] broke down the businessperson's ability to follow up on an open door notwithstanding questionable ecological conditions. They found that accelerations in vulnerability diminished the business visionary's craving to serve [2]. Businesspeople must oversee enterprising danger, objectivity, and significant levels of trouble about business sectors in assessing openings Brown et al. (2001); Miller (2007); Wu \& Knott [3]. Practically speaking, chance inclined business visionaries are encouraged to create methodologies to support unfriendly results Busenitz (1999); Forzani and Mullins (2000). Our emphasis is on assessing the condition of the market for a business opportunity (outside elements, e.g., financial, political, social, and administrative) in view of a blend of business visionary's hazard.

In the assessment stage, business visionaries work under significant vulnerability about the genuine estimation of a chance, and data is expected to evaluate that esteem. This issue in operational business examine by calling for procedures and frameworks to viably catch and use data coming about because of the investigation of potential chances and to improve business visionaries' capacity to refine those potential chances and to follow up on ensuing potential chances. Our paper adds to this writing hole by building up a powerful information driven Markov process for enterprising exercises to assess the market estimation of a perceived possibility while representing the business visionary's hazard inclination and operational deficiencies [4-7].

\section{Proposed System and Implementation}

Our model has empowered us to analyze another technique for assessing a shrouded showcase process, a few suppositions, restrictions, and related augmentations to this exploration should be recognized. To begin with, our key suppositions present inborn confinements on our model. For instance, despite the fact that our 
presumption about an exogenous and free change in the valuation of speculations doesn't really change our bits of knowledge, the unwinding of this suspicion could prompt increasingly significant bits of knowledge into the market. Second, a DP takes into account nonlinearity, way reliance, and unusualness. These properties are significant, accepting that a Markov model is a nearby portrayal of dynamic in true enterprising settings.

In our model the market won't uncover its state legitimately, however, gives related data by means of another procedure detectable by the business visionary. Models of this sort, where the irregular market condition is spoken to by a Markov chain however the condition of this Markov chain can't be watched legitimately (another procedure can give incomplete data about the state), are designated "somewhat watched Markov choice procedure" (POMDP). Our POMDP model sets up a probabilistic connection between a detectable and the shrouded showcase process by means of the business visionary's speculation portfolio. We at that point build up an advancing probabilistic data measure, described by the business visionary's speculation portfolio, to evaluate the association between the recognizable and concealed procedure. We show how the probabilistic data measure examines flawed information about outside elements and thus, encourages the business visionary to comprehend the exchange off between higher expected returns and market data. We further look at numerically how the business visionary's hazard inclination and deficiencies of two of the 4Rs (i.e., inside imperatives) may influence the key exchange off [8-11]. As the information driven point of view of enterprise recommends a unique pioneering process, we build up an algorithmic information investigation procedure for the businesspeople to process flawed information and uncover the concealed idea of the market over a specific timeframe. Outstandingly, every episode of an operational deficiency in our model perceives an alternate level of effect on the business visionary's assessment of the condition of the market for the business opportunity (i.e., to what degree the market bolsters the business opportunity). The level of that effect is additionally directed by the business visionary's individual hazard inclination. Our findings expand the writing on pioneering dynamic and information driven business by analyzing how the businessperson's hazard inclination and operational deficiencies shape the businessperson's chance related assessments and choices in a dynamic and persistently advancing condition. As indicated by our numerical outcomes, the businessperson may pick a speculation portfolio, subject to his/ her degrees of money and hazard inclination, that would permit a hazard supporting or market-controlling technique instead of looking for just high anticipated returns. By and by, our POMDPbased model furnishes business visionaries and supervisors with an algorithmic information examination strategy to utilize bits of knowledge from information investigation for assessing the engaging quality of a business opportunity (Figure 1).

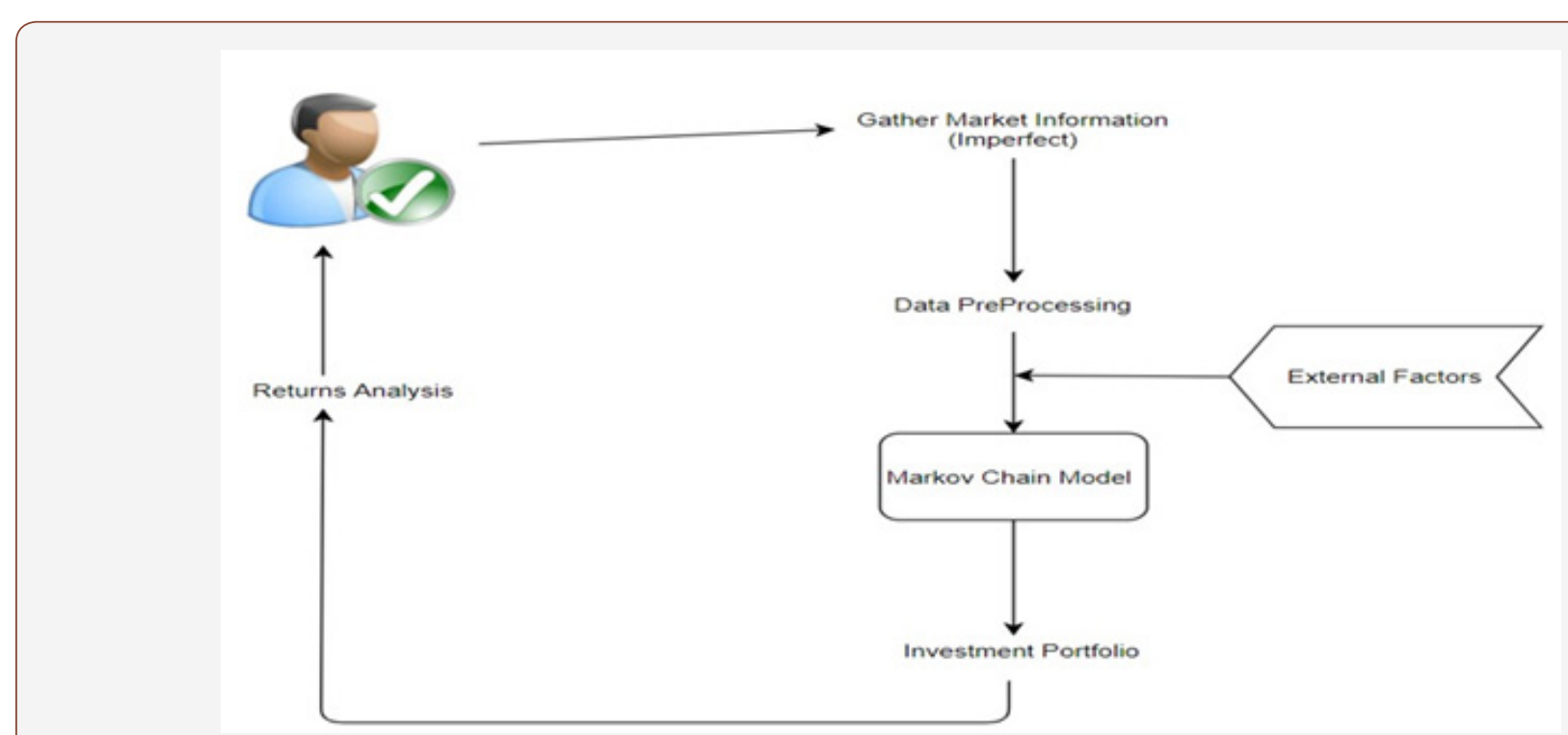

Figure 1: Architecture for Proposed System.

Compositional details are comprehended and structured in this stage. Normally, more than one specialized methodology is proposed, and dependent on the specialized and money related practicality, a ultimate choice is to be taken. Framework configuration is separated further into modules taking up various functionality and is alluded as High-Level Design HLD.
The information moves and correspondence between the interior modules and with the outside world's different frameworks are unmistakably comprehended and characterized in this stage. With this data, incorporation tests can be structured and reported during this stage. 


\section{Experimental Setup and Results}

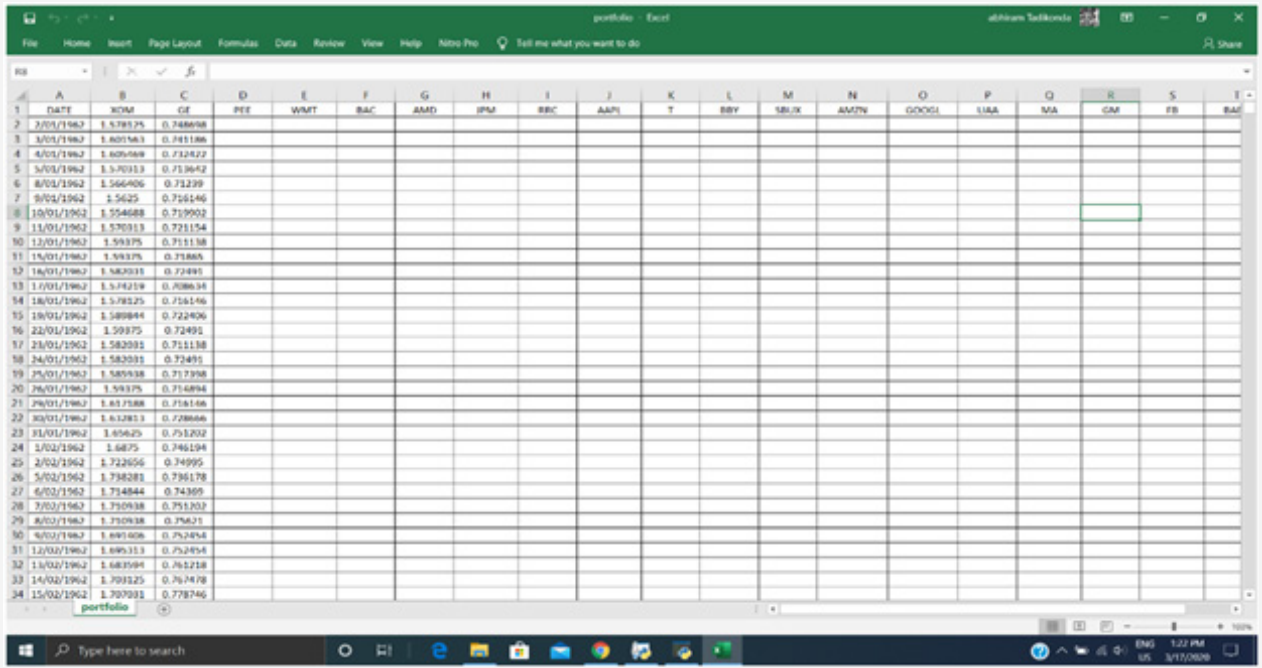

Figure 2: Data Set.

Step 1: Dataset that is inside the portfolio and below are some values from the dataset which is to be taken (Figure 2).

In above dataset all decimal values are the invested amount and XOM is the stock name of ExxonMobil, GE is for General Electric, PEE is for Pfizer Inc., WMT is for Walmart Inc., BAC is for Bank of America Corporation, AMD is for Advanced Micro Devices Inc., JPM is for JMP Group LLC, RRC is for Range Resources Corporation, AAPL is for apple, $\mathrm{T}$ is for AT\&T Inc., BBY is for Best Buy Co Inc., SBUX is for Starbucks Corporation, AMZN is for amazon, GOOG is for google, UAA is for, Under Armor Inc., MA is for MasterCard Incorporated, GM is for General Motors company, FB is for Face Book Inc., BABA is for Alababa Group Holding Limited, SHLD is for Sears Holdings Corporation.

Step2: Double click on 'run.bat' file to get the below screen (Figure 3).

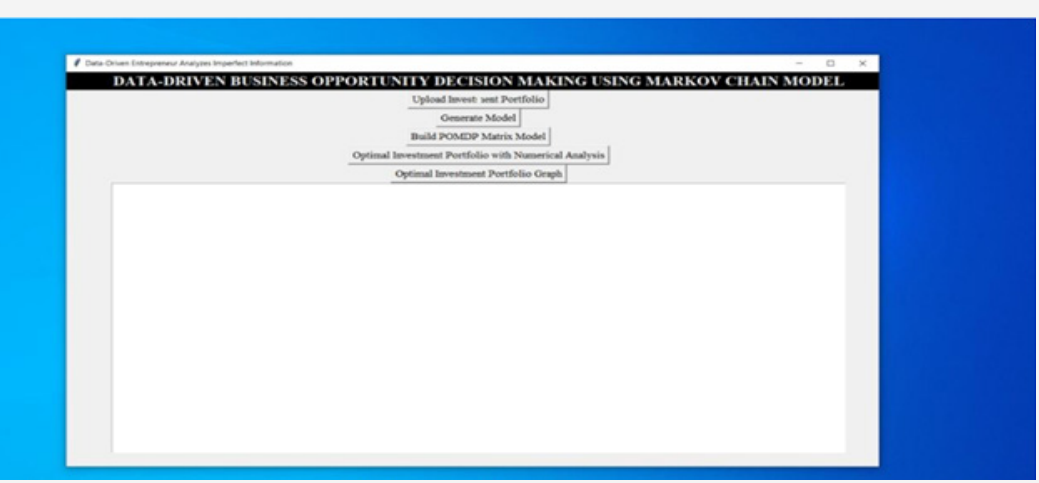

Figure 3: User Interface.

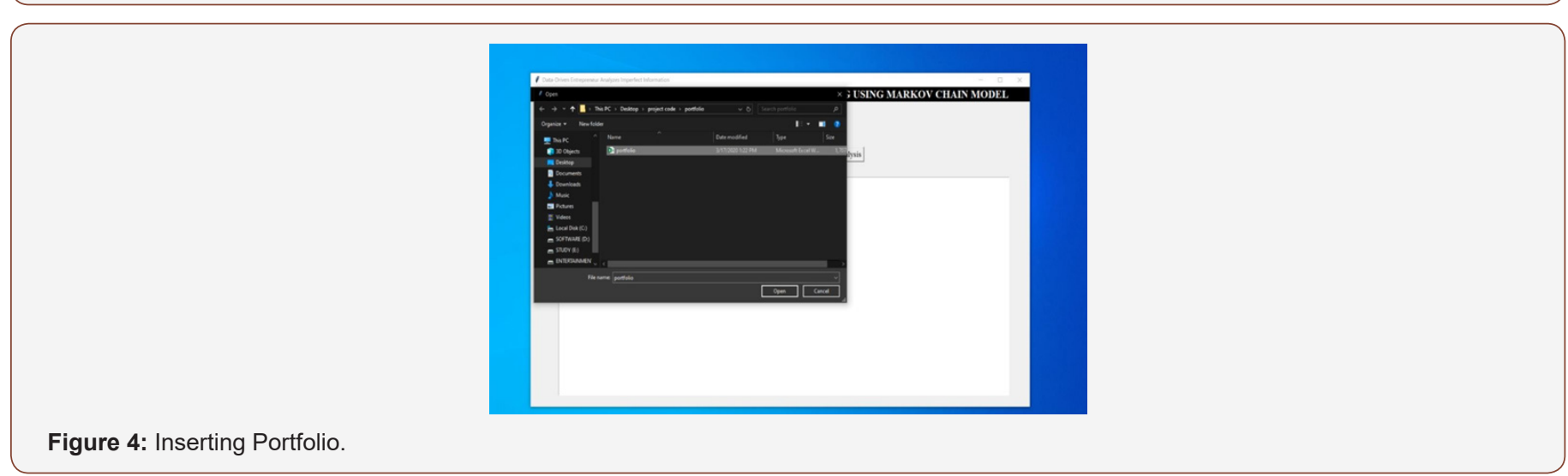


Step 3: Click on the 'Upload Investment Portfolio' button to upload portfolio data (Figure 4).
Step 4: After uploading the portfolio will get the below screen (Figure 5).

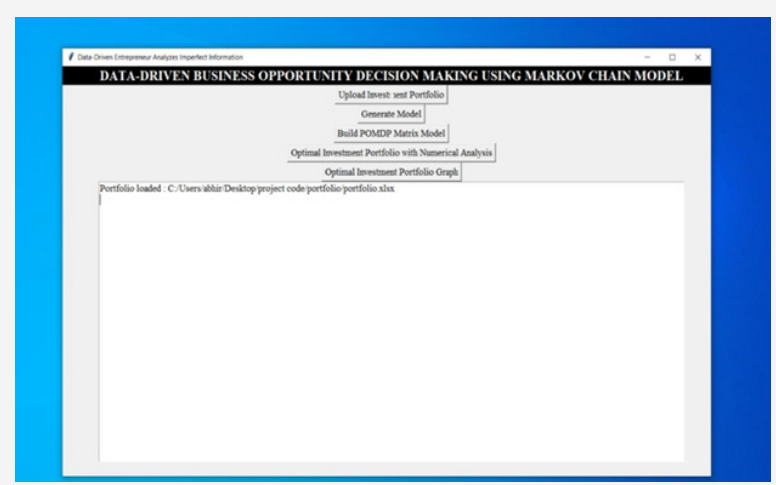

Figure 5: Output after the portfolio insertion.

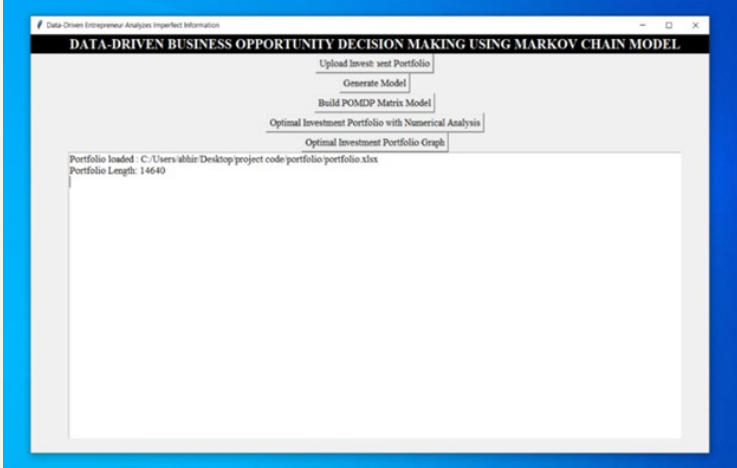

Figure 6: Generating Model.

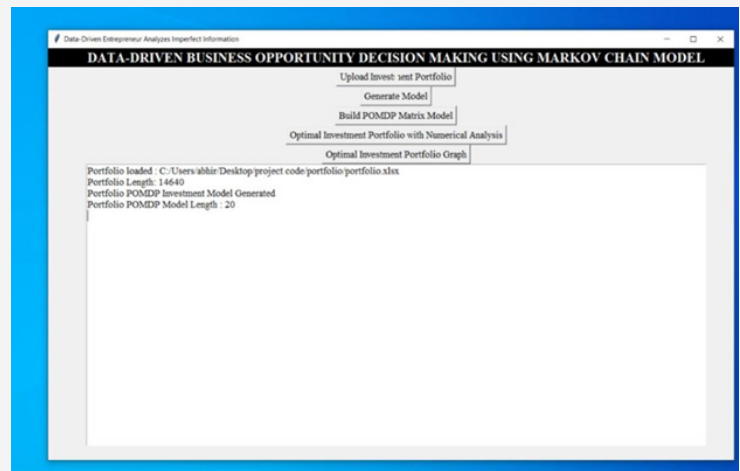

Figure 7: Building the POMDP Matrix Model.

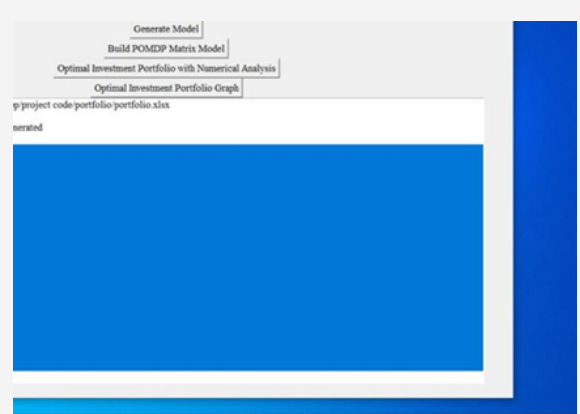

Figure 8: Optimal Investment Portfolio with Numerical Analysis. 
Step 5: Click on 'Generate Model' button to create matrix with Z values from portfolio (Figure 6).

As a result of that portfolio contains total of 14640 records, which contains investment data.

Step6: Click on the 'Build POMDP Model' button to build the POMDP matrix model (Figure 7).

Step 7: Click on 'Optimal Investment Portfolio with Numerical Analysis' to analyze a model to get optimal the portfolio (Figure 8).

As a result of that, some companies have given returns and some returns $0 \%$ profit.

We currently dissect the businessperson's normal returns amplification issue regarding the assessment of a business opportunity with the DP arrangement methodology sketched out in the past area. To get unequivocal portrayals of the ideal speculation portfolio, we define an outflow framework and force utility capacity.
For the discharge lattice, we think about the accompanying example for an assortment of $\mathrm{m}$ speculations and market factor.

Step 8: Now, scroll down to see which company returns high, low, and medium (Figure 9).

As result in POMDP model that MA and FB Company returns high profit and other returns low and medium profit. To clearly see return values follow the next step.

Step 9: Scroll down to view all output of the company wise list (Figure 10).

The total annual returns of the companies show the returns high and low profit. From above screen users can easily understand which company responds high profit and in which they have to invest.

Step 10: Click on 'Optimal Investment Portfolio Graph' button to get the below graph (Figure 11).

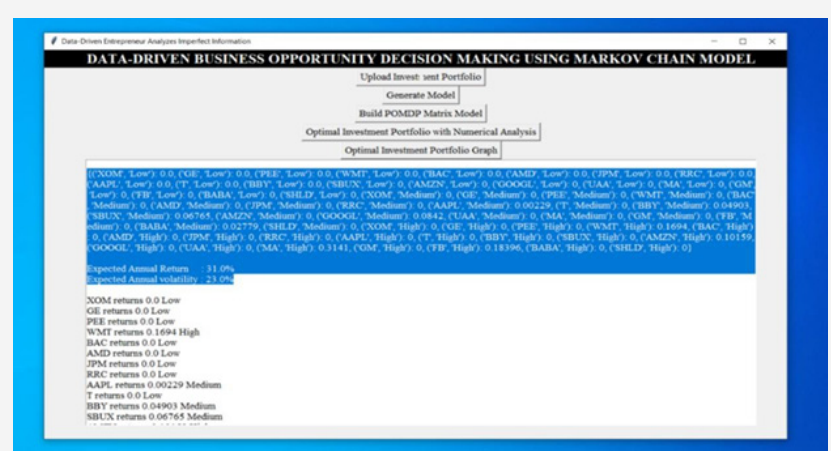

Figure 9: Showing Annual returns and volatility.

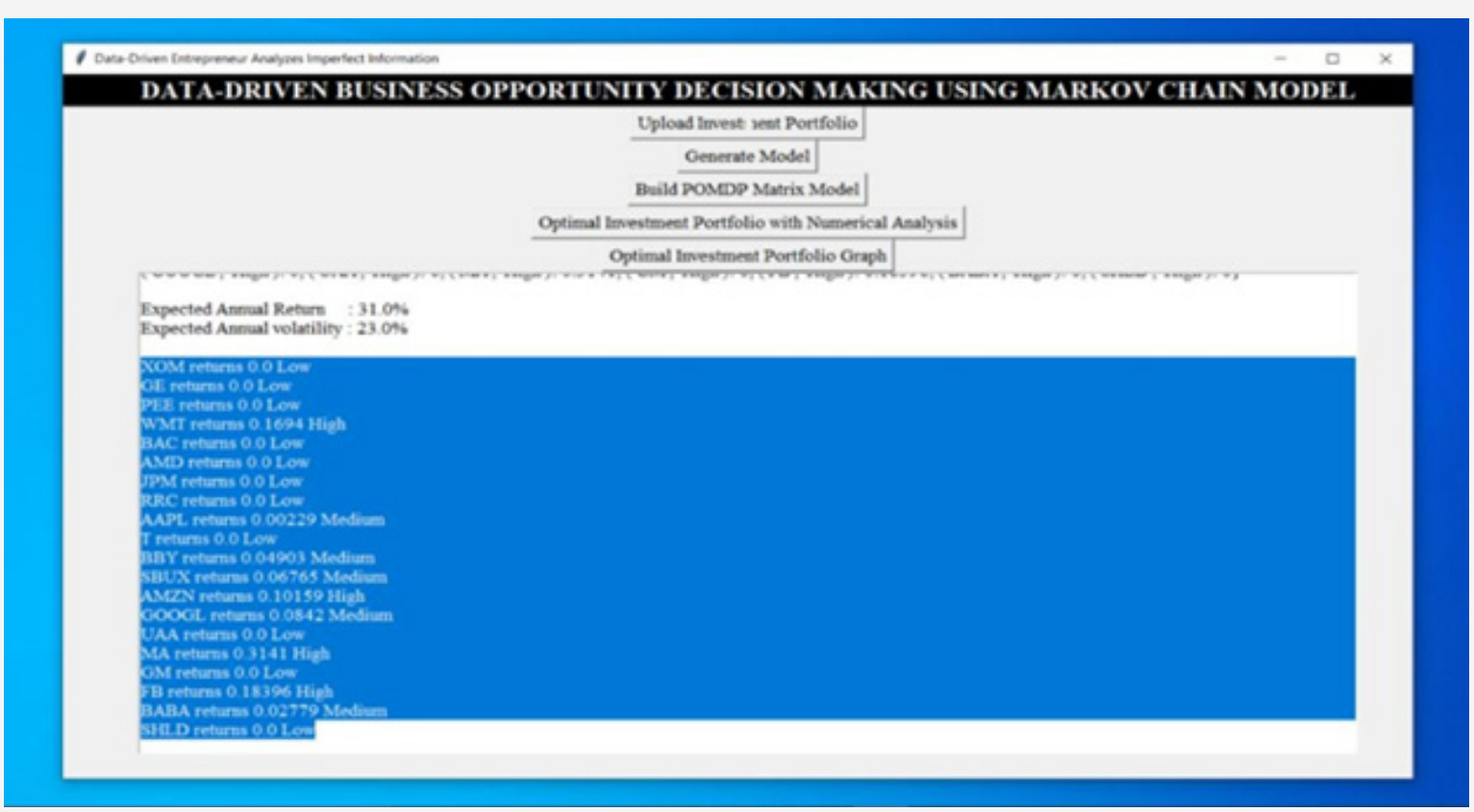

Figure 10: Optimal Investment Portfolio with Numerical Analysis and level of cash. 


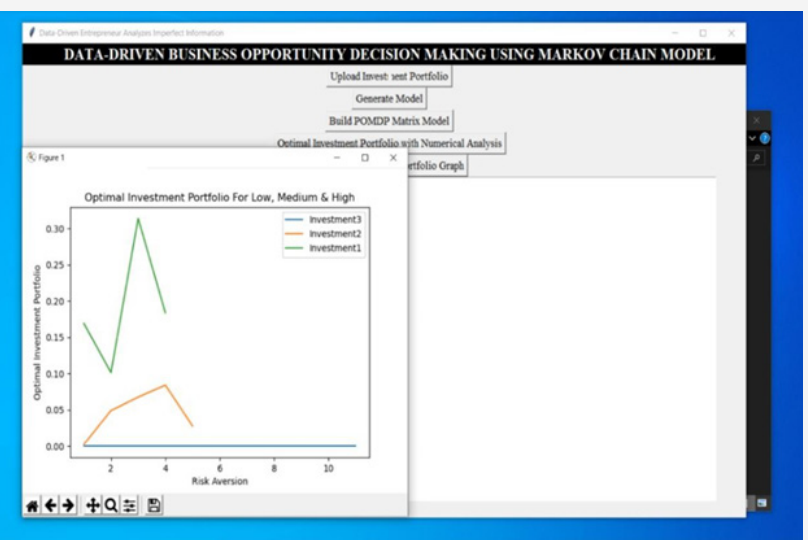

Figure 11: Optimal Investment Portfolio graph.

Graph x-axis represents record no of each investment as Risk Aversion and y-axis represents returns on investment or optimized portfolio value. In the above graph showing 3 investment records where investment1 showing high returns, investment e showing medium gains and investment 3 showing 0 gains. So, by seeing this the users can easily understand and invest in those companies which are returning high profits.

\section{Conclusion}

Attributable to Markovian adjustment of the POMDP model, the discoveries of our dynamic model are more practical than standard static models. Though one can infer a shut structure answer for explicit probabilistic estimates utilizing a POMDP, shut structure systematic articulations can't be acquired for certain cases, for example, circumstances where the speculation dollar sum decides the degree of Information gain. In this manner, we build up a calculation that numerically copies the POMDP-based model. We offer a few bits of knowledge from our numerical investigation because of our subsequent research question on the effect of the business person's inside elements on the exchange off between expected returns and market data: Rather than seeking after the most noteworthy due returns, an information driven business person may pick flawless data, hazard supporting, or market controlling speculations dependent on business visionary money level and hazard inclination in expanding the endeavor's possibilities. For instance, when the deficiency of guidelines or the businessperson's hazard avoidance makes a higher hazard presentation, the business visionary might be progressively effective settling on a hazard supporting speculation choice to relieve that introduction. Specifically, to extend shows that the information driven leader confronted with enterprising vulnerabilities may be increasingly effective by planning a speculation portfolio specifically custom fitted to a noticeable Markovian market and afterward breaking down continuous and defective information for dynamic. In that capacity, the business visionary's resourcefulness, powered by the accessibility of information and diagnostic models, can assume a pivotal job in relieving market vulnerabilities.

\section{Future Work}

Despite the fact that our model has empowered us to painstakingly inspect another strategy to assess a shrouded showcase process, a few suspicions, confinements, and related augmentations to be recognized, powerful programming considers nonlinearity, way reliance, and unusualness. These properties are basic expecting that a Markov model is a nearby portrayal of dynamic in true enterprising settings. Specifically, a dynamic POMDP model could empower the business visionary to utilize his/her financial assets with a speculation portfolio, to process information, and to all the more likely comprehend, evaluate, and advance the conceivable market sway.

\section{Acknowledgement}

None.

\section{Conflict of Interest}

No conflict of interest.

\section{References}

1. A Mc Kelvie, JM Haynie, V Gustavsson (2011) Unloading the vulnerability develop: Implications for enterprising activity. J Transport Wandering 26: 273-292.

2. BG Baykan (2012) Regulation and Financing is High in Renewable Energy News. Pp. 4-6.

3. B Wu, AM Knott (2006) Pioneering danger and market section Manage. Sci 52(9): 1315-1330.

4. Bakker RM, Shepherd DA (2017) Reassess or dive in: Multiple chances and the speed of wandering choices in the Australian mining industry. Under correction.

5. C Gaimon, J Bailey (2013) Information the board for the enterprising endeavor. Prod Oper Oversee 22(6): 1429-1438.

6. Canakoglu E, Ozekici S (2011) Portfolio choice with flawed data: A concealed markov model. Applied Stochastic Models in Business and Industry 27: 95-14.

7. Charness G, Gneexy U, Imas A (2013) Exploratory strategies: Eliciting hazard inclinations. Diary of Economic Behavior and Organization 87: 43-51. 
8. Costa OLV, Araujo MV (2008) A summed up multi-period meanfluctuation portfolio improvement with markov exchanging parameters. Automatica 44: 2487-2497.

9. Data-Driven Entrepreneurship: A Data Analysis Approach to Business Opportunity Evaluation. Ethem Canakoglu Industrial Engineering Department, Bahcesehir University, 34353, Istanbul Turkey.

10. Data-Driven Entrepreneurship: A Data Analysis Approach to Business Opportunity Evaluation. S Sinan Erzurumlu innovation,
Operations and Information Management Division, Babson College, MA 02457, USA, pp. 1-56.

11. Ethem Canakoglu, S Sinan Erzurumlu, Yaman Omer Erzurumlu (2018) How Data-Driven Entrepreneur Analyzes Imperfect Information for Business Opportunity Evaluation. IEEE Transactions on Engineering Management. 